\title{
Áreas marinas protegidas en la alta mar: perspectivas y desafíos en el contexto del derecho internacional
}

\author{
Francisco Gutiérrez Figueroa*
}

\begin{abstract}
RESUMEN
El artículo examina un asunto de reciente interés en el ámbito del derecho internacional: la creación de áreas marinas protegidas en la alta mar. Con ese propósito, el autor analiza la importancia de la conservación marina en la gestión global de los recursos oceánicos, explora los recientes desarrollos vinculados a la creación de áreas marinas protegidas en la alta mar a la luz del ordenamiento jurídico internacional vigente y opina sobre la creciente tendencia de establecer dichas áreas protegidas. Asimismo, el artículo repasa sucintamente los principales instrumentos y procesos internacionales relacionados con la conservación y uso sostenible de la biodiversidad marina en zonas fuera de la jurisdicción nacional.

Palabras clave: Áreas marinas protegidas, alta mar, derecho internacional, derecho del mar, libertad de los mares, biodiversidad marina, conservación, Convención del Mar, gobernanza de los océanos.
\end{abstract}

\section{Marine Protected Areas in the High Seas: prospects and challenges in the context of international law}

\section{Abstract}

The paper reviews a topic of recent interest in the context of international law such as the designation of marine protected areas in the high seas. To that end, the author analyzes the importance of marine conservation in the global management of ocean resources, explores the recent developments related to the designation of marine

\footnotetext{
* Diplomático de carrera. Segundo Secretario en el Servicio Diplomático de la República del Perú, desempeña funciones en la Dirección de Asuntos Marítimos del Ministerio de Relaciones Exteriores. Licenciado en Derecho por la Pontificia Universidad Católica del Perú y máster en Diplomacia y Relaciones Internacionales por la Academia Diplomática del Perú. Diploma de la Academia de Rodas de Derecho y Política Oceánicos (Grecia) y de la Academia de Yeosu de Derecho del Mar (Corea del Sur). Ha realizado estancias de investigación en la División de Asuntos Oceánicos y del Derecho del Mar de las Naciones Unidas (DOALOS) y en el Gerard J. Mangone Center for Marine Policy de la Universidad de Delaware (EE.UU). Correo electrónico: fgutierrezf@pucp.pe.
} 
protected areas in the high seas within the framework of the current international legal order and gives his views on the growing trend of establishing the aforesaid protected areas. Likewise, the paper briefly reviews the main international instruments and processes related to the conservation and sustainable use of marine biodiversity beyond areas of national jurisdiction.

Keywords: Marine protected areas, high seas, international law, law of the sea, freedom of the seas, marine biodiversity, conservation, UNCLOS, ocean governance. 


\section{La conservación marina ayer y hoy: una introducción}

Como demuestra la historia, hasta antes del siglo pasado los recursos de los océanos se acercaron más a la representación de lo infinito que cualquier otro componente del planeta Tierra (Notarbartolo di Sciara, 2015, p. 145). Incluso en el pensamiento de Hugo Grocio - el más célebre defensor de la teoría del mare liberum —, la inapropiabilidad del mar, junto con la inagotabilidad de sus recursos, fueron los fundamentos cardinales para la formulación del principio de la libertad de los mares (Grocio, 1979, pp.108-109,134) ${ }^{1}$. Desde entonces, este principio se ha convertido en una de las piedras angulares del derecho del mar $^{2}$.

Hoy en día sabemos que los océanos y sus recursos están lejos de ser infinitos. La integridad ecológica de los mares se encuentra en peligro como consecuencia de actividades humanas que provocan cambios significativos en todos los espacios oceánicos, desde el mar territorial de los Estados ribereños hasta los fondos marinos y oceánicos fuera de los límites de la jurisdicción nacional. Si esta tendencia nociva persiste, ecosistemas marinos enteros - como ciertos arrecifes de coral tropicalespodrían considerarse desde ya condenados a desaparecer por causa de la sobrepesca, la contaminación marina, el calentamiento global y la acidificación de los océanos (Notarbartolo di Sciara, 2015, p. 145).

La diversidad biológica (en adelante biodiversidad) marina es fundamental para la vida humana debido a que proporciona, directa e indirectamente, servicios ecosistémicos ${ }^{3}$ esenciales que sostienen toda forma de vida en el planeta. Sin embargo, la necesidad de proteger la inmensa variedad de seres vivos marinos y sus ecosistemas apenas emergió en la segunda mitad del siglo XX, una vez establecidos los primeros tratados enfocados en la gestión global de los recursos oceánicos ${ }^{4}$.

\footnotetext{
1 Con relación a la inapropiabilidad del mar, Grocio (1979) afirmaba que «sin duda nada aparece más ajustado a la realidad que lo que afirmaron nuestros Doctores, que el mar, al no poder ser abarcado, lo mismo que el aire, no podía ser agregado a los bienes de pueblo alguno» (pp. 108-109). Con respecto a la inagotabilidad de los recursos marinos, sostenía que «si es manifiesto que muchos individuos cazan en un mismo bosque o pescan en un mismo río, fácilmente se termina con la caza del bosque o con las pesca del río, cosa que no sucede en el mar» (p. 134).

2 Los otros dos principios que rigen el derecho del mar contemporáneo son el de soberanía (el cual busca salvaguardar los intereses del Estado ribereño en el espacio marino adyacente a sus costas) y el de patrimonio común de la humanidad (el cual apunta hacia la promoción de intereses comunitarios bajo un concepto que se sostiene en un eje trans-espacial y en otro trans-temporal). Al respecto, puede consultarse: Tanaka (2015, pp. 16-19); Pureza (2002, p. 234).

3 Siguiendo la definición de la denominada «Evaluación de los Ecosistemas del Milenio» de las Naciones Unidas (Millennium Ecosystem Assesment, 2005), por «servicios ecosistémicos» debe entenderse «the benefits people obtain from ecosystems» (p.v). En el ámbito marino, estos servicios se traducen, por ejemplo, en el aprovisionamiento de alimentos, el aprovechamiento de los recursos genéticos, la regulación del clima, la práctica de actividades recreativas y turísticas, entre otros.

4 En dicho período, las primeras instituciones que surgieron sobre la base de tratados fueron la Organización de las Naciones Unidas para la Alimentación y la Agricultura (FAO) en 1945, dedicada, entre otros asuntos, a la pesca y
} 
En un escenario de ecosistemas y biodiversidad en o al borde del colapso, con un estado mundial de la pesca atravesando situaciones de crisis en varias partes de los mares y océanos del mundo (FAO, 2014, p. 40), y con hábitats marinos cada vez más degradados y disfuncionales, es manifiesto que la conservación de los recursos marinos «is no longer an option [but] a pressing necessity» (Roff y Zacharias, 2011, p. xiv).

La conservación marina puede llevarse a cabo desde varios ángulos. En términos generales, los enfoques de conservación pueden basarse en especies - como, por ejemplo, las medidas generales de conservación de los albatros y petreles-, en espacios - como, por ejemplo, las áreas marinas protegidas-, o en una combinación de ambos - como en el caso del manejo integrado de zonas costeras o la gestión basada en ecosistemas.

En las últimas décadas la conservación marina ha cambiado su foco de atención. En ese sentido, se ha pasado de un enfoque basado en especies - esto es, una perspectiva «tradicional» para conservar el medio marino, considerada por algunos como escasamente efectiva (Proelss y Houghton, 2015, pp. 247-248) — hacia un enfoque holístico, mediante el cual las especies están protegidas en, y junto con, el medio físico en donde habitan (UNEP, 2011, p. 10). Precisamente, las áreas marinas protegidas constituyen una de las expresiones de este último enfoque.

El presente artículo abordará algunos de los aspectos jurídicos relativos al establecimiento de las áreas marinas protegidas, en particular, de aquellas ubicadas en la alta mar. Las recientes discusiones sobre esta materia en diversos procesos internacionales demuestran que aún existen retos y desafíos por superar, sobre todo en lo que respecta a la necesidad de conciliar el tradicional principio de la libertad de los mares con la obligación de conservar la biodiversidad marina en zonas fuera la jurisdicción nacional.

La preocupación de la comunidad internacional por proteger los ecosistemas marinos en beneficio de las actuales y futuras generaciones abre paso a nuevos esquemas de conservación en un espacio marino, como la alta mar, que comprende el $64 \%$ de la superficie total de los mares y océanos del mundo.

La aceptación general de las áreas marinas protegidas en la alta mar reside en la solución a la ecuación conservación-libertad en el contexto del derecho internacional vigente.

\footnotetext{
la acuicultura; y la Organización Marítima Internacional (OMI) en 1948, dedicada a la regulación de la navegación marítima. De igual importancia son los convenios que se adoptaron luego de la Primera Conferencia de las Naciones Unidas sobre el Derecho del Mar de 1958. Con todo, no será hasta 1982, con la adopción de la Convención de las Naciones Unidas sobre el Derecho del Mar, en el que una reglamentación integral y sistemática de los mares y océanos habrá de tener lugar.
} 


\section{Las áreas marinas protegidas: consideraciones generales}

Las áreas marinas protegidas (en adelante AMP) buscan resguardar los ecosistemas marinos en una determinada zona del espacio oceánico, ya sea dentro o fuera de los límites de la jurisdicción nacional. Aunque no existe una definición generalmente aceptada de AMP, se puede considerar la definición de «área protegida» propuesta por la Unión Internacional para la Conservación de la Naturaleza (IUCN por sus siglas en inglés). Según esta definición, que también abarca las áreas de naturaleza marina, un «área protegida» es:

Un espacio geográfico claramente definido, reconocido, dedicado y gestionado, mediante medios legales u otros tipos de medios eficaces para conseguir la conservación a largo plazo de la naturaleza y de sus servicios ecosistémicos y sus valores culturales asociados (Dudley, 2008, p.10).

Las AMP constituyen una herramienta moderna en la gestión integral de los océanos. En efecto, en los últimos 40 años $^{5}$ el concepto de protección del medio ambiente marino ha trasladado su punto de mira, en un tránsito de la prevención y control de la contaminación hacia nociones más amplias de manejo espacial e integrado de los ecosistemas marinos (Scott, 2012, pp. 849-850). A diferencia de las medidas de conservación basadas en categorías específicas de especies marinas, las AMP buscan proteger las especies, hábitats y ecosistemas marinos de manera integrada considerando la intrínseca relación existente entre ellos.

En las últimas tres décadas la noción de AMP ha ido evolucionando y actualmente abarca una amplia gama de propósitos que incluye, entre otros, la protección de ecosistemas vulnerables y el desarrollo de capacidades de recuperación frente a los efectos de la navegación, la sobrepesca y el cambio climático (Scott, 2012, p. 850). Asimismo, las AMP proporcionan distintos niveles de protección y uso bajo muy variados esquemas de gestión. Así pues, mientras algunas permiten actividades recreacionales o comerciales y actividades pesqueras, otras prohíben ciertas actividades extractivas tales como la perforación de pozos petroleros y gasíferos (Connolly, 2015, p. 594). Por lo tanto, es seguro afirmar que cada AMP representa un caso particular.

La Convención de las Naciones Unidas sobre el Derecho del Mar (en adelante CONVEMAR) no ofrece ninguna referencia acerca del concepto de AMP. Sin embargo, en el ámbito de la Parte XII (Protección y Preservación del Medio Marino),

\footnotetext{
5 Aunque los tratados que posibilitan la creación de AMP datan de la década de 1940 (como es el caso de la Convención para la Protección de la Naturaleza y la Vida Silvestre en el Hemisferio Occidental, adoptada en 1940 y en vigor desde 1942), es en la década de 1970 cuando se empieza a prestar atención a los conceptos jurídicos relacionados con las AMP. Al respecto, vid. Tanaka (2015, pp. 346-347).
} 
los Estados se encuentran en la obligación de preservar y proteger el medio marino (CONVEMAR, artículo192). Específicamente, la CONVEMAR dispone que los Estados adopten las medidas que sean necesarias para "proteger y preservar los ecosistemas raros o vulnerables, así como el hábitat de las especies y otras formas de vida marina diezmadas, amenazadas o en peligro" (artículo 194.5). Y, por último, establece la obligación de cooperar, en el plano mundial y regional, en la protección y preservación del medio marino considerando las características propias de cada región (artículo 197).

Por otro lado, el Convenio sobre la Diversidad Biológica (en adelante CDB) ${ }^{6}$, cuyo ámbito de aplicación también se extiende a los espacios marinos, determina que un «área protegida» es un «área definida geográficamente que haya sido designada o regulada y administrada a fin de alcanzar objetivos específicos de conservación» (CDB, artículo 2). El CDB también dispone que cada Parte Contratante, «en la medida de lo posible y según proceda», establecerá un sistema de áreas protegidas o áreas donde haya que tomar medidas especiales para conservar la biodiversidad (art. 8.a). Con todo, una lectura sistemática de los artículos 4 y 22.2 del $\mathrm{CDB}^{7}$ sugiere que las áreas protegidas, a la luz de ese tratado, solamente pueden ser creadas dentro de los espacios marinos bajo jurisdicción de las partes contratantes.

Hoy en día existen cerca de cinco mil AMP, gran parte de ellas ubicadas en las zonas de jurisdicción de los Estados ribereńos. En total, las AMP cubren un área aproximada de 2,85 millones de kilómetros cuadrados, lo que equivale a casi 1\% del área total de los mares y océanos del mundo (De Sombre y Barkin, 2011, p. 108). Entre las mayores AMP (en términos de extensión) se encuentran el de las Islas Fénix (Kiribati) con un área total de 410500 kilómetros cuadrados, el de la Gran Barrera de Coral (Australia) con 344400 kilómetros cuadrados y el de Papahānaumokuākea (Estados Unidos) con 341400 kilómetros cuadrados (IUCN-Protect Planet Ocean, 2011). Para diciembre de 2017, el Mar de Ross (Antártida), con 1,5 millones de kilómetros cuadrados, se convertirá en el área protegida de mayor extensión en el mundo.

\footnotetext{
6 El CDB fue adoptado en la Conferencia de las Naciones Unidas sobre el Medio Ambiente y el Desarrollo, llevada a cabo en Río de Janeiro en 1992. Es el primer tratado que aborda de modo exhaustivo la conservación de la biodiversidad. Por lo tanto, no se limita solamente a la protección de algunas especies y hábitats como había ocurrido con instrumentos anteriores.

7 El artículo 4 del CDB señala que «con sujeción a los derechos de otros Estados, y a menos que se establezca expresamente otra cosa en el presente Convenio, las disposiciones del Convenio se aplicarán, en relación con cada Parte Contratante: a) En el caso de componentes de la diversidad biológica, en las zonas situadas dentro de los límites de su jurisdicción nacional; y b) En el caso de procesos y actividades realizados bajo su jurisdicción o control, y con independencia de dónde se manifiesten sus efectos, dentro o fuera de las zonas sujetas a su jurisdicción nacional». Por su parte, el artículo 22.2 menciona que «las Partes Contratantes aplicarán el presente Convenio con respecto al medio marino, de conformidad con los derechos y obligaciones de los Estados con arreglo al derecho del mar».
} 
Se reconoce ampliamente a las AMP como herramientas efectivas para la protección y conservación de los recursos marinos vivos. Las AMP benefician a una serie de especies marinas, entre las que se incluyen peces, tortugas, tiburones y mamíferos. Estas áreas potencian la capacidad reproductiva de las especies, mantienen la biodiversidad marina, preservan tanto los hábitats como las funciones ecosistémicas, y sostienen la producción pesquera (Connolly, 2015, p. 595).

Sobre la pesca en particular, las AMP han demostrado tener efectos beneficiosos sobre las especies que se reproducen o se refugian en ellas. De hecho, se ha comprobado que ciertas AMP han favorecido el incremento de la biomasa total en el área y la recuperación de especies pesqueras agotadas. De igual manera, al mejorar la biodiversidad marina en general (en términos de cantidad y de calidad), se contribuye con la optimización del rendimiento de las especies de interés comercial, así como de aquellas que no son objetivo de la pesca (De Sombre y Barkin, 2011, p. 106).

A pesar de todas estas ventajas, la creación de una AMP no constituye per se la solución a todos los problemas relacionados con las actividades humanas sobre el medio marino. En efecto, se puede afirmar que ellas son «a testimony of our inability to properly manage human activities at sea, as there would be little need for them in a scenario of diffused, effective sustainability» (Notarbartolo di Sciara, 2015, p. 147).

En cualquier caso, para que la puesta en marcha de una AMP resulte satisfactoria se requerirá, en primer lugar, la armonización de aspectos ecológicos, sociales, políticos - y también jurídicos-, en el marco de un proyecto de conservación marina. En segundo lugar, el éxito de una AMP dependerá en gran medida de dos componentes fundamentales: creación oportuna y gestión eficaz (Crofts, 2017, p. xii). Por último, será necesario coordinar los esfuerzos desplegados por las AMP — particularmente a nivel regional - con el fin de hacer frente a las diversas amenazas que enfrentan los océanos. A modo de ejemplo, si la sobrepesca no se reduce a escala global, las actividades de pesca se trasladarán a áreas donde no existen regulaciones eficaces y, en consecuencia, las AMP devendrían contribuciones necesarias pero insuficientes a la administración sostenible de los recursos marinos.

Como es evidente, la cooperación internacional juega un rol trascendental en la búsqueda de soluciones a los problemas que enfrenta la comunidad internacional en la conservación y uso sostenible de la biodiversidad marina, requerimiento que es aún más urgente en el ámbito del régimen jurídico de la alta mar. 


\section{Las áreas marinas protegidas en la alta mar: instrumentos y procesos internacionales}

La necesidad de establecer AMP en la alta mar es un asunto que ha estado presente en recientes procesos e instrumentos internacionales, en los cuales estas son percibidas como herramientas de soporte al concepto de desarrollo sostenible. En la Agenda 21 - el plan de acción aprobado en la Conferencia de las Naciones Unidas sobre el Medio Ambiente y el Desarrollo de 1992 (Cumbre de Río) — ya se invocaba a los Estados a proteger y reponer las especies marinas en peligro, y a conservar los hábitats y otras zonas ecológicamente expuestas de la alta mar (UNEP, 1992, párrs. 17.46,e y 17.46,f); del mismo modo que se resaltaba la importancia de la cooperación internacional para "promover el mejoramiento de la reunión de los datos necesarios para la conservación y el aprovechamiento sostenible de los recursos marinos vivos de la alta mar»(párr. 17.56,a).

En el Plan de Aplicación de las Decisiones de la Cumbre Mundial sobre el Desarrollo Sostenible de 2002 (Cumbre de Johannesburgo) se reafirmaba la necesidad de conservación y ordenación de los océanos para «mantener la productividad y la diversidad biológica de las zonas marinas y costeras importantes y vulnerables, incluidas las zonas situadas fuera de la jurisdicción nacional», así como para «elaborar diversos enfoques e instrumentos y facilitar su uso, incluido [...] el establecimiento de zonas marinas protegidas de conformidad con el derecho internacional y sobre la base de información científica» (WSSD, 2002, párrs. 32, a y 32, c).

En el informe sobre el Proceso Abierto de Consultas Oficiosas de las Naciones Unidas sobre los Océanos y el Derecho del Mar (UNICPOLOS o ICP por sus siglas en inglés) de 2003, se dejó constancia del apoyo mostrado por muchas delegaciones al establecimiento de las AMP como instrumento de ordenación integrada de los océanos, incluidas las zonas fuera de los límites de la jurisdicción nacional. Específicamente, sobre las AMP en la alta mar:

[A] lgunas delegaciones subrayaron que esas zonas protegidas debían: i) basarse en pruebas científicas, ii) poder hacerse cumplir, iii) responder a las características y objetivos concretos de cada zona, iv) ser compatible con el criterio del ecosistema, y v) ser conformes con el derecho internacional. (Naciones Unidas, 2003, párr. 104)

Por otro lado, en la Octava Conferencia de las Partes (COP) del CDB de 2006, se reconoció que las AMP «son una de las herramientas esenciales para lograr la conservación y utilización sostenible de la diversidad biológica en áreas marinas fuera de la jurisdicción nacional» (UNEP, 2006, párr. 38). En esa línea también se enmarcó la decisión de dicho órgano de formular, en 2008, criterios científicos para identificar Áreas Marinas de Importancia Ecológica o Biológica (EBSA por sus siglas en inglés) 
que requieren protección en aguas oceánicas abiertas y en hábitats de aguas profundas (UNEP, 2008, pp. 7-11). Complementariamente, la Décima COP del CBD de 2010 estableció las denominadas «Metas de Aichi para la Diversidad Bilógica», cuya meta 11 propone que, para el año 2020, al menos el 10 por ciento de la zonas marinas y costeras se conserven "por medio de sistemas de áreas protegidas administrados de manera eficaz y equitativa, ecológicamente representativos y bien conectados» (UNEP, 2010, p. 11) ${ }^{8}$.

La cuestión del establecimiento de AMP en la alta mar también estuvo presente en los debates del Grupo de Trabajo Especial Oficioso de Composición Abierta encargado de estudiar las cuestiones relativas a la conservación y el uso sostenible de la diversidad biológica marina fuera de las zonas de jurisdicción nacional (en adelante Grupo de Trabajo Especial) ${ }^{9}$. Concretamente, en 2011 el Grupo de Trabajo Especial recomendó a la Asamblea General de las Naciones Unidas que inicie un proceso destinado a desarrollar un régimen jurídico aplicable a la biodiversidad marina en zonas fuera de la jurisdicción nacional, incluida la posibilidad de elaborar un tratado multilateral en el marco de la CONVEMAR. Las AMP fueron destacadas en las recomendaciones de ese foro, dentro de los cuatro grandes temas identificados en el proceso encaminado a la conservación y uso sostenible de la biodiversidad marina en zonas fuera de la jurisdicción nacional ${ }^{10}$.

De igual modo, en el documento titulado El futuro que queremos, emanado de la Conferencia de las Naciones Unidas sobre el Desarrollo Sostenible de 2012 (Cumbre de Río +20), los Estados se comprometieron a abordar con urgencia el asunto de la conservación y uso sostenible de la biodiversidad marina en zonas fuera de la jurisdicción nacional (Naciones Unidas, 2012, párr.162). Concretamente, se reafirmó la importancia de las AMP, en cuanto medidas de conservación basadas en zonas geográficas, como instrumento para lograr esos objetivos (párr. 177).

Volviendo a las actividades del Grupo de Trabajo Especial, debe resaltarse que aquel enfoque integrado y temático promovido en 2011 ha sido característico de sus reuniones ulteriores, así como de las resoluciones conexas de la Asamblea General

\footnotetext{
8 Cabe resaltar un informe de la Secretaría del CDB (2014) en el que menciona que actualmente la meta 11 de las "Metas de Aichi para la Diversidad Biológica» no se encuentra adecuadamente cubierta con respecto a las zonas de alta mar y aguas profundas (p.14).

9 El Grupo de Trabajo Especial fue establecido en virtud del párrafo 73 de la resolución 59/24 de la Asamblea General de Naciones Unidas de 17 de noviembre de 2004. La primera reunión de este grupo de trabajo se llevó a cabo del 13 al 17 de febrero 2006 y la última, del 20 al 23 de enero de 2015. En total se celebraron nueve reuniones. 10 Los otros tres temas, que en conjunto componen el denominado "paquete de 2011», son los siguientes: recursos genéticos marinos, incluidas cuestiones relativas a la participación en los beneficios; evaluaciones del impacto ambiental; y creación de capacidad y transferencia de tecnología marina. Conviene aclarar que en este contexto las AMP han sido consideradas como parte de los mecanismos de ordenación basados en zonas geográficas.
} 
de las Naciones Unidas ${ }^{11}$. Con la finalidad de centrar el análisis en el tema principal de este artículo, resulta interesante apreciar el sentir de los Estados con relación a la conveniencia de establecer AMP en la alta mar. Al respecto, en 2014, durante el desarrollo del penúltimo de los encuentros del Grupo de Trabajo Especial:

Varias delegaciones expresaron su apoyo a la elaboración de un régimen o mecanismo de gobernanza mundial que permitiera establecer zonas marinas protegidas en la alta mar mediante un proceso intergubernamental legítimo, fundado en criterios científicos. Muchas delegaciones hicieron hincapié en que las zonas marinas protegidas no podían establecerse en zonas fuera de la jurisdicción nacional de manera unilateral, en ausencia de un tratado o acuerdo mundial, y que un instrumento internacional, por ende, aseguraría la legitimidad. (Naciones Unidas, 2014, párr. 63)

Una vez finalizadas las funciones del Grupo de Trabajo Especial en 2015, la Asamblea General de las Naciones Unidas decidió establecer ese mismo año, antes de que se celebre una conferencia intergubernamental para elaborar un instrumento internacional relativo a la conservación y uso sostenible de la biodiversidad marina en zonas fuera de la jurisdicción nacional, un Comité Preparatorio abierto a todos los Estados miembros de las Naciones Unidas - y a otros actores involucrados en la materia-, para que formule recomendaciones sustantivas a la Asamblea General sobre los elementos de un proyecto de texto de un instrumento internacional jurídicamente vinculante teniendo en cuenta los diversos informes del Grupo de Trabajo Especial (Naciones Unidas, 2015, párr. 1.a) ${ }^{12}$. En este nuevo ámbito de debates en torno a la biodiversidad marina en zonas fuera de la jurisdicción nacional, se decidió también que los cuatros temas que conforman el "paquete de 2011» sean tratados en las negociaciones con vistas a un futuro tratado.

En lo que respecta al establecimiento de AMP en la alta mar, las dos primeras sesiones del Comité Preparatorio de $2016^{13}$ aparentemente mostraron una coincidencia

11 Como mencionan Long y Rodríguez (2015) con respecto al "paquete de 2011», «it ought to be mentioned that the consensus and integrated approach is not unusual and reflects the unique circumstances from which the 2011 package deal emerged and will ensure that each element of the negotiations is given equal weight, with a view to engendering compromise and universal acceptance of the outcome of the process. Indeed, this approach is typical of law of the sea negotiations since the Third United Nations Conference on the Law of the Sea (1973-1982) and has contributed to the extraordinary achievements and almost universal acceptance of UNCLOS over the past three decades» (p. 219).

12 Conforme a dicha resolución (párrafo 1.b), el Comité Preparatorio sesionará en dos ocasiones durante el año 2016 y otras dos durante el año 2017. Asimismo, y aunque parezca obvio, el mismo instrumento resalta que ni el Comité Preparatorio ni la futura conferencia intergubernamental deben «ir en detrimento de los instrumentos y marcos jurídicos existentes ni de los órganos globales, regionales y sectoriales competentes» (párrafo 3). Por último, la Asamblea General adoptará una decisión sobre la convocatoria y fecha de inicio de la conferencia intergubernamental antes de que concluya el septuagésimo segundo período de sesiones de ese órgano de las Naciones Unidas (párrafo 1.k).

13 La primera y segunda sesiones del Comité Preparatorio se desarrollaron del 28 de marzo al 8 de abril de 2016 y del 26 de agosto al 9 de setiembre de 2016, respectivamente. 
de puntos de vista acerca de la obligación estatal de proteger y preservar el medio marino (en concordancia con el artículo 192 de la CONVEMAR) y la relevancia de los mecanismos de gestión basados en zonas geográficas, incluyendo las AMP, para la conservación y el uso sostenible de la biodiversidad marina en zonas fuera de la jurisdicción nacional. También parece haber existido convergencia en el respeto a los derechos del Estado ribereńo sobre su plataforma continental, incluso en los casos en que esta se extiende más allá de las 200 millas marinas ${ }^{14}$.

Sin embargo, en el ámbito de dicho proceso también existieron puntos de divergencia en aspectos mucho más cruciales, tales como la definición de conceptos relacionados a los mecanismos de ordenación basados en zonas geográficas; el establecimiento de algún aparato institucional (de alcance global o regional) en apoyo a la creación de AMP en la alta mar; la determinación de un proceso de toma de decisiones; y la aplicación de ciertos principios o enfoques, especialmente en lo que corresponde, por un lado, al balance entre conservación y uso sostenible y, por otro lado, al balance entre las libertades de la alta mar y la obligación general de protección y preservación del medio marino.

Uno de los desafíos para la cuarta y última sesión del Comité Preparatorio (prevista para julio de 2017) será el de formular recomendaciones específicas sobre los mecanismos de gestión basados en zonas geográficas y la creación de AMP en la alta mar. Además de encontrar una solución a los puntos de divergencia anteriormente mencionados, también será necesario identificar estrategias para compatibilizar el funcionamiento de las AMP en la alta mar con las medidas adoptadas por el Estado ribereño adyacente, así como seleccionar el enfoque de gobernanza más apropiado (es decir, un modelo global, regional o híbrido).

\section{Las áreas marinas protegidas en la alta mar: algunas consideraciones desde la óptica del derecho internacional}

Aunque en el derecho internacional no existe ninguna norma que prohíba la creación de AMP en la alta mar, también es cierto que no existe una base jurídica a nivel multilateral que permita su establecimiento. En ausencia de tal marco jurídico, la conciliación de intereses asociados con la conservación del medio ambiente marino y las libertades tradicionales de la alta mar, como la pesca y la navegación, representa hoy en día un desafío en la ordenación jurídica de los océanos. Ciertamente, la

\footnotetext{
${ }_{14} \mathrm{Al}$ respecto, se puede revisar en detalle los documentos que contienen la visión de conjunto del presidente del Comité Preparatorio sobre las dos primeras sesiones desde los siguientes enlaces: http://www.un.org/depts/ los/biodiversity/prepcom_files/PrepCom_1_Chair's_Overview.pdf; http://www.un.org/depts/los/biodiversity/ prepcom_files/Prep_Com_II_Chair_overview_to_MS.pdf
} 
protección de los intereses comunitarios está tomando cada vez mayor importancia en el derecho internacional general, situación que también se refleja en el ámbito del derecho del mar. $\mathrm{Al}$ respecto, Tanaka resalta esta apreciación al enfatizar que:

Given its vital importance for the survival of mankind, it could well be said that conservation of marine biological diversity is considered as a common interest of the international community as a whole. Thus, legal issues respecting conservation of marine biological diversity, including marine protected areas, must be examined in the context of the protection of community interests at sea (Tanaka, 2012, p. 297).

En este nuevo escenario, la práctica de ciertos Estados parece apoyar la creación de AMP en la alta mar. Así es como algunas de estas áreas han sido cerradas a la pesca en general o a ciertos tipos de actividades pesqueras (como por ejemplo la pesca de arrastre) bajo el auspicio de diversas Organizaciones Regionales de Ordenamiento Pesquero (en adelante OROP) ${ }^{15}$.

El caso más significativo de esta tendencia es el de la Comisión para la Conservación de los Recursos Vivos Marinos Antárticos (CCAMLR) pues se trata de la primera organización regional en establecer un área protegida ubicada enteramente en la alta mar. En 2009 esta OROP estableció una AMP cerca de las islas Orcadas del Sur (South Orkney) en el Océano Antártico. De acuerdo con las medidas adoptadas por la CCAMLR, en dicha AMP, de 94000 kilómetros cuadrados de extensión, todo tipo de actividad pesquera se encuentra prohibida (con excepción de la que se realice con fines científicos), así como también el vertido de residuos por parte de cualquier embarcación pesquera (CCAMLR, 2009). Por su parte, el AMP del Mar de Ross, con una extensión de 1.5 millones de kilómetros cuadrados, iniciará su funcionamiento a partir de diciembre de 2017 — también por iniciativa de la CCAMLR—, e impondrá similares restricciones a las actividades humanas en la alta mar con el fin de satisfacer objetivos específicos de conservación, vigilancia de los ecosistemas y ordenación pesquera (CCAMLR, 2016).

Por otro lado, entre 2010 y 2012 la Comisión OSPAR ${ }^{16}$ estableció una red de siete AMP en la zona de la alta mar del Atlántico Nordeste ${ }^{17}$. Este grupo de AMP, que cubre

\footnotetext{
15 Entre estas OROP se cuentan la Comisión de Pesca del Atlántico Nordeste (NEAFC), la Organización de Pesquerías del Atlántico Noroccidental (NAFO), la Organización de Pesquerías del Atlántico Suroriental (SEAFO), la Comisión General de Pesca para el Mediterráneo (GFCM), la Comisión para la Conservación de los Recursos Vivos Marinos Antárticos (CCAMLR), entre otras.

16 OSPAR (denominación derivada de las Convenciones de Oslo y París) es el mecanismo por el cual 15 Estados europeos (Alemania, Bélgica, Dinamarca, España, Finlandia, Francia, Irlanda, Islandia, Luxemburgo, Países Bajos, Noruega, Portugal, Reino Unido, Suecia y Suiza) y la Unión Europea cooperan en la protección del medio ambiente marino del Atlántico Nordeste. La Convención OSPAR fue adoptada el 22 de setiembre de 1992 y entró en vigor el 25 de marzo de 1998. Su principal órgano es la Comisión OSPAR, cuya Secretaría se encuentra en Londres.

17 Estas AMP son las siguientes: Milne Seamount Complex, Charlie Gibbs South, Altair Seamount, Antialtair Seamount, Josephine Seamount, Mid Atlantic Ridge North of the Azores y Charlie-Gibbs North.
} 
más de 386000 kilómetros cuadrados, busca principalmente proteger los hábitats y ecosistemas marinos y prevenir la pérdida de biodiversidad. La Comisión OSPAR ha celebrado diversos Memorándum de Entendimiento con algunas OROP ${ }^{18}$ y otras organizaciones $^{19}$ con el fin de abordar un amplio rango de actividades que tienen lugar dentro o alrededor de esta red de AMP.

La tendencia creciente de establecer AMP en la alta mar merece algunas consideraciones a la luz del Derecho Internacional contemporáneo. En primer lugar, aunque el propósito de ciertas OROP de actuar en interés de la comunidad internacional constituye un avance positivo con relación a la protección y conservación de la biodiversidad marina en zonas fuera de la jurisdicción nacional, el estatus legal de las AMP en la alta mar se encuentra sujeto a cuestionamientos en ausencia de un marco jurídico que sustente su creación en dicho ámbito geográfico. Es cierto que los Estados — por vía convencional y consuetudinaria- se encuentran obligados, por un lado, a proteger y preservar el medio marino $\mathrm{o}^{20} \mathrm{y}$, por otro lado, a cooperar entre sí en la conservación y administración de los recursos vivos de la alta mar ${ }^{21}$; empero, es discutible que estas obligaciones generales otorguen a los Estados el derecho de establecer AMP más allá de los límites de la jurisdicción nacional (Tanaka, 2015, p. 353). Por el momento no existe una justificación jurídica sólida para defender la constitución de dichas áreas protegidas en la alta mar.

En segundo lugar, la solución a la cuestión de la legitimidad de las AMP en la alta mar es un prerrequisito indispensable para conseguir el incremento de su aceptación internacional. Dado que no existe una organización internacional de alcance global para la conservación y uso sostenible de la biodiversidad en la alta mar, podría admitirse que las organizaciones regionales han asumido la tarea de disponer medidas de conservación dentro de sus áreas geográficas de competencia. Sin embargo, aun aceptando esta idea, tales medidas de conservación no serían aplicables para los buques que enarbolen el pabellón de un Estado que no es miembro de dichas organizaciones internacionales. De igual manera, al no ser parte del proceso de toma de decisiones para determinar la ubicación de las AMP en la alta mar, los Estados no partes del acuerdo o mecanismo regional podrían cuestionar la legitimidad de dicho proceso. Frente a ello, es necesario establecer procedimientos que aseguren la participación de todos los actores con intereses en los espacios marinos en cuestión junto con procesos

\footnotetext{
18 Por ejemplo, con la Organización para la Conservación del Salmón del Atlántico Norte (NASCO) y la NEAFC.

19 Por ejemplo, con la Autoridad Internacional de los Fondos Marinos, el Consejo Internacional para la Exploración del Mar (CIEM), la Agencia Europea del Medio Ambiente (AEMA), entre otras. Con la Organización Marítima Internacional (OMI) se celebró un acuerdo de cooperación.

20 En concordancia con los artículos 192 y 194.5 de la CONVEMAR.

21 En concordancia con los artículos 117 y 118 de la CONVEMAR.
} 
de toma de decisiones transparentes e información científica relevante para la selección de potenciales AMP en la alta mar (Tanaka, 2012, pp. 317-318).

En tercer lugar, la creación de AMP en la alta mar podría encontrar cierta incompatibilidad con el principio de la libertad de los mares. Sobre el particular, el artículo 237.2 de la CONVEMAR refiere que «las obligaciones específicas contraídas por los Estados en virtud de convenciones especiales con respecto a la protección y preservación del medio marino deben cumplirse de manera compatible con los principios y objetivos generales de esta Convención». Y además el artículo 311.2 enfatiza que:

Esta Convención no modificará los derechos ni las obligaciones de los Estados Partes dimanantes de otros acuerdos compatibles con ella y que no afecten al disfrute de los derechos ni al cumplimiento de las obligaciones que a los demás Estados Partes correspondan en virtud de la Convención.

Es indudable que la libertad de los mares es uno de los principios esenciales de la CONVEMAR (y del derecho del mar en general). En ese sentido, sería válido aseverar que las medidas regulatorias adoptadas por las organizaciones regionales que designan AMP en la alta mar no deberían afectar el disfrute de los derechos de otros Estados conforme al principio de la libertad de los mares. Precisamente, la creación de AMP podría implicar el riesgo de limitar, además de la libertad de pesca, las libertades de navegación, de tendido de cables y tuberías submarinos, de construcción de islas artificiales, y de investigación científica. En tal escenario, los Estados afectados por dichas limitaciones difícilmente aceptarían el uso de las AMP como herramientas de manejo integrado de los ecosistemas marinos. Por ejemplo, en los debates dentro de la Comisión OSPAR para el establecimiento de la red de siete AMP en la alta mar, la tensión permanente entre los conceptos de conservación y uso racional de los recursos vivos marinos estuvo y continúa estando latente (Scott, 2012, p. 855). La misma circunstancia puede apreciarse en el contexto de los debates del Grupo de Trabajo Especial y, más recientemente, en el marco del Comité Preparatorio relativo a la biodiversidad marina en zonas fuera de la jurisdicción nacional. Como se puede inferir de lo anteriormente expuesto, los asuntos concernientes a las libertades de pesca y navegación tendrían que ser abordados dentro del marco funcional de las AMP con el fin de lograr un balance sostenible y evitar su ineficacia ab initio.

Por último, pero no menos importante, mientras las regulaciones de las AMP en la alta mar son aplicables solamente a la columna de agua ubicada más allá de los límites de la jurisdicción nacional, debajo de esta rigen — según sea el caso- las disposiciones de la Parte XI de la CONVEMAR, relativas a la Zona (de los fondos marinos y oceánicos) o las de la Parte VI, vinculadas al régimen jurídico de la plataforma continental extendida. En tales condiciones es necesario que la organización regional que establezca la AMP asegure la coordinación intersectorial en favor de la conservación 
integral de la biodiversidad marina, ya sea con la Autoridad Internacional de los Fondos Marinos (en adelante la Autoridad) ${ }^{22}$, ya sea con el Estado ribereńo con derechos de soberanía sobre una plataforma continental extendida.

La creación de AMP en estas circunstancias es perfectamente posible, tal como lo demuestra la cooperación entre Portugal y la Comisión OSPAR con respecto a la red de AMP de esta última. En efecto, cuatro de las AMP establecidas por la Comisión OSPAR $^{23}$ se ubican en las aguas suprayacentes dentro de los límites de una plataforma continental extendida reclamada por Portugal ${ }^{24}$. Tomando como muestra representativa una de las recomendaciones emitidas por la Comisión OSPAR, esta reconoce que:

[T] he Portuguese Republic has reported to the OSPAR Commission on the selection of the seabed of the Altair Seamount as a component of the OSPAR network of Marine Protected Areas and will establish the programmes, measures and agreements which are necessary for the achievement of the conservation vision and conservation objectives regarding the seabed of the Altair Seamount and [welcomes] the request of the Portuguese Republic to the OSPAR Commission to take corresponding measures for the waters superjacent to the Altair Seamount. (OSPAR, 2010, Preámbulo, párr. 10)

Adicionalmente, la recomendación señala que la Comisión OSPAR puede tomar las acciones necesarias con el fin de lograr la conservación de las aguas de la alta mar suprayacentes a la plataforma continental reclamada por Portugal (párr. 2.1), lo que otorga coherencia, compatibilidad y complementariedad a las medidas de gestión adoptadas en y más allá de los límites de la jurisdicción nacional.

Asimismo, en 2008 la Comisión OSPAR y la Comisión de Pesca del Atlántico Nordeste (NEAFC) adoptaron un Memorándum de Entendimiento con el objetivo de asegurar la coordinación y cooperación en vista de que ambas organizaciones regionales tienen competencias complementarias en lo que respecta a la protección del ambiente y la ordenación pesquera, respectivamente, en el Atlántico Nordeste, incluyendo las zonas fuera de la jurisdicción nacional. En ese sentido, ambas partes acordaron promover la conservación y uso sostenible de la biodiversidad marina en el Atlántico Nordeste a través de la cooperación internacional en áreas tales como el

\footnotetext{
22 De acuerdo con la CONVEMAR (artículo 157.1), la Autoridad Internacional de los Fondos Marinos es la organización por medio de la cual los Estados partes organizan y controlan las actividades de los fondos marinos y oceánicos y su subsuelo con miras a la administración de los recursos minerales de ese espacio marino fuera de la jurisdicción nacional.

23 Altair Seamount, Antialtair Seamount, Josephine Seamount y Mid Atlantic Ridge North of the Azores.

24 El ámbito espacial de la plataforma continental más allá de las 200 millas marinas reclamada por Portugal aún no ha sido determinado. El límite exterior de la plataforma continental será determinado por Portugal sobre la base de las recomendaciones de la Comisión de Límites de la Plataforma Continental, de acuerdo con las disposiciones que para estos casos establece la CONVEMAR (en particular el artículo 76 y el Anexo II de ese instrumento internacional).
} 
intercambio de información; la gestión de actividades humanas con impacto sobre el ambiente marino y los recursos marinos vivos, así como posibles medidas para abordarlas; la aplicación del principio o enfoque precautorio; entre otras (OSPAR, 2008, párr. 1). La cooperación entre la Comisión OSPAR y la NEAFC resulta significativa pues las áreas de competencia de ambas organizaciones se superponen completamente en la zona de la alta mar del Atlántico Nordeste ${ }^{25}$.

De igual manera, en 2010 la Comisión OSPAR celebró un Memorándum de Entendimiento con la Autoridad a fin de cooperar en la coordinación de acciones que permitan conciliar el desarrollo de actividades vinculadas con los recursos minerales de la Zona y la protección integral del ambiente marino. De acuerdo con lo expresado, ambas partes acordaron, inter alia, fomentar la investigación científica en las áreas marinas del Atlántico Nordeste ubicadas fuera de la jurisdicción nacional, y cooperar en la recolección de información y datos ambientales (OSPAR, 2010, párrs. 2 y 3$)$.

La Autoridad está llamada a jugar un rol importante en la protección y conservación ambiental de la Zona, tal como lo dispone el artículo 145 de la CONVEMAR, el cual dispone que esa entidad establecerá las normas, reglamentos y procedimientos apropiados para, entre otras cosas:

a. Prevenir, reducir y controlar la contaminación del medio marino y otros riesgos para este, incluidas las costas, y la perturbación del equilibrio ecológico del medio marino, prestando especial atención a la necesidad de protección contra las consecuencias nocivas de actividades tales como la perforación, el dragado, la excavación, la evacuación de desechos, la construcción y el funcionamiento o mantenimiento de instalaciones, tuberías y otros dispositivos relacionados con tales actividades;

b. Proteger y conservar los recursos naturales de la Zona y prevenir daños a la flora y fauna marinas.

Es preciso resaltar que la jurisdicción de la Autoridad no se extiende a la columna de agua suprayacente a la Zona. Su jurisdicción se limita a los asuntos contenidos en la Parte XI de la CONVEMAR y en el Acuerdo relativo a la aplicación de la Parte XI de 1994. En tal escenario, por ejemplo, al encontrarse la regulación de las pesquerías y la contaminación marina en la alta mar fuera del ámbito de la Autoridad, se requeriría asegurar la cooperación entre la Autoridad y las OROP (u otro tipo de

\footnotetext{
25 Ambas organizaciones tienen competencias distintas pero complementarias. La Comisión OSPAR tiene el mandato de regular actividades humanas que pueden afectar negativamente el mar, con excepción de la pesca y la navegación. Por su parte, la NEAFC tiene el mandato de procurar la conservación y el uso óptimo de los recursos pesqueros.
} 
organizaciones internacionales) con el fin de conservar y utilizar sosteniblemente la biodiversidad marina bajo un enfoque integrado. En ese orden de ideas, la ya mencionada cooperación entre la Autoridad y la Comisión OSPAR resulta ser largamente positiva.

La conservación y administración de los recursos vivos de la alta mar plantea un nuevo desafío para los Estados y requiere la aplicación del principio de cooperación internacional y voluntad política para impulsar una mejor gobernanza de los océanos (O’Leary et al., 2012, p. 603). En ambos aspectos, no se debería dejar de lado el papel de la buena fe - entendida como una verdadera obligación jurídica que corresponde a todos los Estados - en las discusiones y negociaciones acerca de cómo abordar las amenazas y riesgos a los que se encuentra sometida la biodiversidad marina en zonas fuera de la jurisdicción nacional (Scovazzi, 2004, p. 6).

A juzgar por los recientes procesos internacionales sobre la materia que han tenido lugar en los últimos años, un régimen jurídico para las AMP en la alta mar se encuentra inevitablemente en proceso de germinación. La actual ausencia de un marco multilateral que provea de una base jurídica específica para la creación de estas áreas protegidas limita su expansión como elementos de manejo integrado de los ecosistemas marinos. Será necesario, por lo tanto, contar con principios y mecanismos institucionales concretos capaces de reconciliar, de un lado, el interés general por la conservación del medio marino y, de otro lado, el interés de los Estados ribereños y las organizaciones internacionales con competencias en la alta mar.

\section{El principio de la libertad de los mares en el siglo XXI: reflexiones finales}

La tesis defensora del principio de la libertad de los mares, sistematizada por Hugo Grocio en el siglo XVII, logró consolidarse a lo largo del siglo XVIII gracias, por un lado, a los cambios en las relaciones de poder económico y militar y, por otro lado, al respaldo de una mayor cantidad de tratadistas sobre la base de argumentos sólidos; todo lo cual influyó decisivamente en favor de esa construcción jurídica en la práctica estatal posterior.

Precisamente, diversos acontecimientos que irrumpieron a partir del siglo XVIII (tales como la represión de la trata de esclavos y el contrabando) dotaron de un nuevo significado a las actividades emprendidas por los Estados en el mar y, con ello, a una reconfiguración del principio de la libertad de los mares. De hecho, el desarrollo de nuevas técnicas pesqueras favoreció el aumento vertiginoso de la captura de ciertas especias marinas apreciadas por su valor económico, como el caso, entre otras, de las ballenas azul y gris, cuya caza indiscriminada originó en el siglo XIX un serio peligro 
de extinción al superar los límites de sostenibilidad, circunstancia que ocasionó un descenso cuantioso en las poblaciones de estos y otros cetáceos. A raíz de ello, los propios Estados, conscientes de la importancia de la industria pesquera y de los recursos vivos que proporcionaba el mar, fueron imponiendo restricciones a la libertad de pesca mediante el establecimiento de zonas reservadas a comienzos del siglo XX.

Para mediados del siglo XX, en estrecha vinculación con el paulatino desarrollo científico y tecnológico, la investigación de los mares y sus fondos vendría a constituir un factor determinante en la búsqueda de una nueva ordenación jurídica de los mares y océanos.

Todo lo mencionado nos lleva a concluir que el derecho del mar está sujeto a un proceso de evolución y desarrollo progresivo que está vinculado a las nuevas necesidades y a la subsiguiente práctica internacional de los Estados. O expresado de otro modo, "we cannot today use the same concepts that Grotius used four centuries ago and give them the same intellectual and legal strength that Grotius gave them» (Scovazzi, 2004, p. 7).

En ese entendido, se advierte que el ordenamiento jurídico de la alta mar viene perfilándose hacia un régimen que conlleva cada vez mayores restricciones a las libertades tradicionales en ese espacio marino, debido a la aparición de nuevos fenómenos e intereses en el derecho del mar contemporáneo, tales como la jurisdicción rampante reivindicada por ciertos Estados ribereños; la pesca ilegal, no declarada y no reglamentada; el creciente protagonismo de las OROP en la gestión colectiva de los recursos pesqueros; y el tema principal de este artículo: la creación de AMP en la alta mar.

Las limitaciones a las libertades de la alta mar no necesariamente deben considerarse como un perjuicio para los Estados, sino más bien como una preocupación de la comunidad internacional por asegurar una gestión integral colectiva de conservación y administración de los recursos naturales en beneficio de los usuarios presentes y futuros de los mares y océanos, en el marco del amplio concepto del desarrollo sostenible.

Aunque las AMP significan una herramienta esencial para contribuir a la conservación y uso sostenible de la biodiversidad marina en la alta mar, aún no existe una base jurídica internacional concreta que fundamente su creación en zonas fuera de la jurisdicción nacional. Esta circunstancia mantiene en discusión su legitimidad en el marco del derecho internacional contemporáneo.

La negociación de un futuro tratado en el marco de las Naciones Unidas relativo a la conservación y uso sostenible de la biodiversidad marina - proceso que se encuentra actualmente en curso- posiblemente ofrezca una solución a la ecuación 
conservación-libertad en el plano de las relaciones interestatales sobre los océanos. Mientras tanto, es preciso reconocer que las libertades de la alta mar no son absolutas, sino que han de ser ejercidas teniendo debidamente en cuenta los intereses de otros Estados, y más recientemente, los de la comunidad internacional en conjunto.

\section{Referencias}

CCAMLR (2009). Protection of the South Orkney Islands southern shelf(Conservation Measure 91-03).

CCAMLR (2016). Ross Sea region marine protected area (Conservation Measure 91-05).

CDB (Convenio sobre la Diversidad Biológica) (1992).

CDB, Secretaría del Convenio (2014). Perspectiva Mundial sobre la Diversidad Biológica 4: Evaluación a mitad de periodo sobre los avances en la implementación del Plan Estratégico para la Diversidad Biológica 2011-2020. Montreal: Secretaría del Convenio sobre la Diversidad Biológica.

Connolly, K. D. (2015). Marine Protected Areas. En D.C. Baur, T. Eichenberg, G.H. Snusz y M. Sutton (eds.). Ocean and Coastal Law and Policy (pp.593-625). Segunda edición. Chicago: American Bar Association Publishing.

CONVEMAR - Convención de las Naciones Unidas sobre el Derecho del Mar (1982).

Crofts, R. Foreword (2017). En P.D. Goriup (ed.). Management of Marine Protected Areas: A Network Perspective. Chichester: Wiley-Blackwell.

De Sombre, E. R. y J. S. Barkin (2011). Fish. Cambridge: Polity Press.

Dudley, N. (ed.) (2008). Directrices para la aplicación de las categorias de gestión de áreas protegidas. Gland: UICN. https://doi.org/10.2305/IUCN.CH.2008.PAPS.2.es

FAO (2014). El estado mundial de la pesca y la acuicultura. Roma: FAO.

Grocio, H. (1979). De la Libertad de los Mares. Traducción de Vicente Blanco García y Luis García Arias. Madrid: Centro de Estudios Constitucionales.

IUCN-ProtectPlanet Ocean (2011). GlobalfactsaboutMPAsandmarine reserves. Recuperadode http://www.protectplanetocean.org/collections/introduction/introbox/globalmpas/ introductionitem.html

Long, R. y M. Rodríguez Chaves (2015). Anatomy of a new international instrument for marine biodiversity beyond national jurisdiction. First impressions of the preparatory process. Environmental Liability - Law, Policy and Practice, 23 (6).

Millennium Ecosystem Assessment (2005). Ecosystems and Human Well-being: Synthesis. Washington, D.C.: Island Press.

Naciones Unidas (2003). Informe sobre el Proceso Abierto de Consultas Oficiosas de las Naciones Unidas sobre los Océanos y el Derecho del Mar (A/58/95, 26 de junio de 2003).

Naciones Unidas (2012). El futuro que queremos (A/RES/66/288, 27 de julio de 2012).

Naciones Unidas (2014). Resumen de los debates en el Grupo de trabajo Especial Oficioso de Composición Abierta encargado de estudiar las cuestiones relativas a la conservación y el 
uso sostenible de la diversidad biológica marina fuera de las zonas de jurisdicción nacional preparado por los Copresidentes (A/69/177, 23 de julio de 2014).

Naciones Unidas (2015). Elaboración de un instrumento internacional juridicamente vinculante en el marco de la Convención de las Naciones Unidas sobre el Derecho del Mar relativo a la conservación y el uso sostenible de la diversidad biológica marina de las zonas situadas fuera de la jurisdicción nacional (A/RES/69/292, 6 de julio de 2015).

Notarbartolo di Sciara, G. (2015). Marine Conservation. En H. Smith, D. Hance, J.L. Suárez de Vivero y T.S. Agardy (eds.), Routledge Handbook of Ocean Resources and Management. Londres-Nueva York: Routledge.

O’Leary, B.C, Brown, R.L., Johnson, D.E., von Nordheim, H., Ardron J., Packeiser, T., Roberts, C.M. (2012). The first network of marine protected areas (MPAs) in the high seas: The process, the challenges and where next. Marine Policy, 36 (3). https:// doi.org/10.1016/j.marpol.2011.11.003

OSPAR (2008). Memorandum of Understanding between the North East Atlantic Fisheries Commission (NEAFC) and the OSPAR Commission (Agreement 2008-4).

OSPAR (2010). Memorandum of Understanding with the International Seabed Authority (Agreement 2010-9).

OSPAR (2010). OSPAR Recommendation 2010/14 on the Management of the Altair Seamount High Seas Marine Protected Area.

Proelss, A. y K. Houghton (2015). Protecting Marine Species. En R. Rayfuse, (ed.), Research Handbook on International Marine Environmental Law. Cheltenham-Northampton: Edward Elgar. https://doi.org/10.4337/9781781004777.00021

Pureza, J.M. (2002). El Patrimonio Común de la Humanidad. ¿Hacia un Derecho Internacional de la Solidaridad? Madrid: Trotta.

Roff, J. y M. Zacharias (2011). Marine Conservation Ecology. Londres-Nueva York: Earthscan. https://doi.org/10.4324/9781849775540

Scott, K. N. (2012). Conservation on the High Seas: Developing the Concept of the High Seas Marine Protected Areas. The International Journal of Marine and Coastal Law, 27. https://doi.org/10.1163/15718085-12341243

Scovazzi, T. (2004). Marine Protected Areas on the High Seas: Some legal and Policy Considerations. The International Journal of Marine and Coastal Law, 19(1). https:// doi.org/10.1163/157180804773788646

Tanaka, Y. (2012). Reflections on High Seas Marine Protected Areas: A Comparative Analysis of the Mediterranean and the North-East Atlantic Models. Nordic Journal of International Law, 81 (3). https://doi.org/10.1163/15718107-08103004

Tanaka, Y. (2015). The International Law of the Sea. $2^{\circ}$ edición. Cambridge: Cambridge University Press.

UNEP (1992). Agenda 21: Programa de Acción de las Naciones Unidas de Río. Nueva York: Naciones Unidas.

UNEP (2006). Decision adopted by the Conference of the Parties to the Convention on Biological Diversity at its Eighth Meeting (UNEP/CBD/COP/DEC/VIII/24, 15 de junio de 2006). 
UNEP (2008). Decisión adoptada por la Conferencia de las Partes en el Convenio sobre la Diversidad Biológica en su Novena Reunión (UNEP/CBD/COP/DEC/IX/20, 9 de octubre de 2008).

UNEP (2010). Decisión adoptada por la Conferencia de las Partes en el Convenio sobre la Diversidad Biológica en su Décima Reunión (UNEP/CBD/COP/DEC/X/2, 27 de octubre de 2010).

UNEP (2011). Taking Steps toward Marine and Coastal Ecosystem-Based Management. An Introductory Guide. Nairobi: UNEP.

WSSD (2002). Plan de Aplicación de las Decisiones de la Cumbre Mundial sobre el Desarrollo Sostenible. Nueva York: Naciones Unidas.

Fecha de recepción: 19 de mayo de 2017 Fecha de aprobación: 22 de junio de 2017 